DAMTP/94-38

NSF-ITP-94-52

\title{
SUMMING OVER WORLD-SHEET BOUNDARIES
}

\author{
Michael B. Green, \\ DAMTP, Silver Street, Cambridge CB3 9EW, UK ${ }^{\star}$ \\ Joseph Polchinski \\ ITP, University of California, Santa Barbara, CA 93111, USA ${ }^{\dagger}$
}

\begin{abstract}
The moduli associated with boundaries in a Riemann surface are parametrized by the positions and strengths of electric charges. This suggests a method for summing over orientable Riemann surfaces with Dirichlet boundary conditions on the embedding coordinates. A light-cone parameterization of such boundaries is also discussed.
\end{abstract}

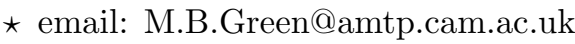

† email: joep@sbitp.ucsb.edu 
The inclusion of boundaries in the sum over world-sheets that defines string perturbation theory alters the properties of string theory rather dramatically. With Neumann boundary conditions on the embedding coordinates $X^{\mu}(\sigma, \tau)$, the resulting theory describes interacting open and closed strings - a boundary representing the trajectory of an open-string end-point. Boundaries with Dirichlet conditions on the embedding coordinates $\left(X^{\mu}(\sigma, \tau)=y^{\mu}\right)$ also have significant effects - for some discussion see [1] and references therein. From the target space point of view, the Dirichlet boundary is simply a point. This suggests using world-sheet coordinates in which the boundary is mapped to a point; what distinguishes this point is singular behavior of the intrinsic metric $g_{a b}$. Since the world-sheet now appears to be topologically trivial, the sum over arbitrary numbers of Dirichlet boundaries can at least formally be recast as a world-sheet field theory, which is the motivation for this paper.

The strategy to be followed is to reexpress the general metric for a surface (of euclidean signature) with an arbitrary number of holes, $\tilde{g}$, as the sum of a metric on a surface with no holes, $g$, and a bilinear in a vector field living on the surface,

$$
\tilde{g}_{\alpha \beta}=g_{\alpha \beta}+A_{\alpha} A_{\beta},
$$

$(\alpha, \beta=1,2)$ where $A=-* d \phi$ (in components, $\left.A_{\alpha}=-g_{\alpha \beta} \epsilon^{\beta \gamma} \partial_{\gamma} \phi\right)$ is a 1 -form vector field. We will refer to $\tilde{g}$ in the following as the 'modified' metric. It bears an obvious resemblence to a metric that would arise in Kaluza-Klein reduction from a three-dimensional theory (in which $g_{\alpha 3}=A_{\alpha}$ ). The inverse modified metric is given by $\tilde{g}^{\alpha \beta}=g^{\alpha \beta}-A^{\alpha} A^{\beta}\left(1+A^{\gamma} A_{\gamma}\right)^{-1}$, where indices are here raised by $g^{\alpha \beta}$ (the inverse of $g$ ). The magnetic field strength due to the vector field is taken to vanish everywhere except at $B$ arbitrary points,

$$
* F \equiv * d A=-d * d \phi=\sqrt{g} \nabla^{2} \phi=\sum_{i=1}^{B} 2 \pi \alpha_{i} \delta^{2}\left(w-w_{i}\right),
$$

where $\alpha_{i}$ are arbitrary real parameters (constrained so that $\sum_{i=1}^{B} \alpha_{i}=0$ ) - in other words, there are $B$ point magnetic vortices on the surface at complex positions $w_{i}$. Equivalently, $\phi$ is the electrostatic potential for $B$ electric charges on the surface. The electric field is given by $E_{\alpha}=\nabla_{\alpha} \phi=g_{\alpha \beta} \epsilon^{\beta \gamma} A_{\gamma}$.

These charges produce the desired effect. Close to a charge of strength $\alpha$, located for convenience at the origin, the vector potential can be written in complex coordinates as

$$
A_{w} d w=-i \partial_{w} \phi d w \sim-i \frac{\alpha}{2} \frac{1}{w} d w=-i \frac{\alpha}{2}\left(\frac{d r}{r}+i d \theta\right),
$$

where $w=r e^{i \theta}$ (and $\left.A_{\bar{w}}=\bar{A}_{w}\right)$. In polar coordinates,

$$
A_{r} \equiv \frac{1}{r}\left(w A_{w}+\bar{w} A_{\bar{w}}\right) \sim 0, \quad A_{\theta} \equiv i\left(w A_{w}-\bar{w} A_{\bar{w}}\right) \sim \alpha .
$$

Therefore, near $r=0, \tilde{g}_{\theta \theta} \sim \alpha^{2}$ and the modified metric, $d \tilde{s}^{2} \sim d r^{2}+\alpha^{2} d \theta^{2}$, is that of the end of a cylinder where $r$ is the coordinate along the axis and the circumference is $2 \pi \alpha$. 
The addition of the $A_{\alpha} A_{\beta}$ term in (1) has converted the metric to one appropriate to a surface with extra boundaries. The parameters of $A_{\alpha}$ determine the complex structure on the surface. The complex position of each additional charge and its strength $\alpha$ are the three parameters that define the moduli of each additional boundary so that the inclusion of $B$ charges of arbitrary strength plausibly defines metrics that cover the whole of moduli space for a surface with $B$ boundaries. The sum over boundaries can be replaced by a functional integral over $\phi$ on a world-sheet with no boundaries.

If $X^{\mu}$ are scalar 'matter' fields on the world-sheet (such as the usual string spacetime coordinates) then $X^{\mu}\left(w_{i}\right)$ (where $w=w_{i}$ is the point at which a charge resides on the original world-sheet) becomes the boundary value of $X^{\mu}$ in the description in which the world-sheet metric is modified and there is a boundary at $w_{i}$. In other words, Dirichlet boundary conditions arise naturally, together with the prescription that the boundary value should be integrated (since $X^{\mu}(w)$ is integrated for all values of $w$ ).

The hope, therefore, is that there is an effective string theory including the vector field that defines the full effect of summing over arbitrary numbers of boundaries on a surface with an arbitrary number of handles. Of course, there remains the usual problem of summing over the number of handles, or genus, of the surface.

\section{Two boundaries}

As a simple explicit example consider first the line element of the constant curvature sphere in stereographic coordinates,

$$
d s^{2}=\frac{1}{\left(1+r^{2}\right)^{2}}\left(d r^{2}+r^{2} d \theta^{2}\right)=\frac{1}{(1+w \bar{w})^{2}} d w d \bar{w}
$$

where $w=r e^{i \theta}, 0 \leq r \leq \infty$ and $0 \leq \theta \leq 2 \pi$. The $r^{2}$ behaviour of $g_{\theta \theta}$ near the origin indicates the coordinate singularity that makes the origin no different from any other point. Now consider the modified metric with two charges of compensating strengths $\alpha$ and $-\alpha$ at the North and South poles (the total charge vanishes by Gauss' law since $A_{\alpha}$ is globally defined). The modified spherical metric is

$$
d \tilde{s}^{2}=\frac{1}{\left(1+r^{2}\right)^{2}}\left(d r^{2}+\left(r^{2}+\alpha^{2}\left(1+r^{2}\right)^{2}\right) d \theta^{2}\right)
$$

Equation (6) defines a metric on a cylinder and can be rewritten in a conformally flat manner by the reparameterization $r \rightarrow r^{\prime}$ defined by

$$
r^{\prime}=\int_{0}^{r} \frac{d r^{\prime \prime}}{\left(r^{\prime \prime 2}+\alpha^{2}\left(1+r^{\prime \prime 2}\right)^{2}\right)^{\frac{1}{2}}}
$$

so that

$$
d \tilde{s}^{2}=\frac{r^{2}+\alpha^{2}\left(1+r^{2}\right)^{2}}{\left(1+r^{2}\right)^{2}}\left(d r^{\prime 2}+d \theta^{2}\right)
$$

In this form the metric is manifestly conformally equivalent to the flat metric on cylinder 
of circumference $2 \pi$ and of length

$$
L(\alpha) \equiv r^{\prime}(r=\infty)-r^{\prime}(r=0)=\int_{0}^{\infty} \frac{d r^{\prime \prime}}{\left(r^{\prime \prime 2}+\alpha^{2}\left(1+r^{\prime \prime 2}\right)^{2}\right)^{\frac{1}{2}}}
$$

which is finite for finite $\alpha$. The boundaries of moduli space are given by $L(\alpha \rightarrow \infty) \rightarrow 0$ and $L(\alpha \rightarrow 0) \sim-\ln \alpha \rightarrow \infty$. It is easy to see that the Euler character of the surface vanishes as expected from the Gauss-Bonnet theorem. More generally, the two charges may be at asymmetric positions $\left(w_{1}\right.$ and $\left.w_{2},\right)$ on the initial sphere but this gives a modified metric in the same conformal class as the above.

\section{Multi-boundary insertions in a disk}

The connection between the parameters of the charges and the moduli of the surface is obscured by the fact that the action of the Mobius group, $S L(2, C)$, on (1) is not a conformal transformation. Indeed, if it were, the number of independent real parameters describing a sphere with $B$ charges would be $3 B-7$ (taking the constraint $\sum_{i=1}^{B} \alpha_{i}$ into account), which is one fewer than the number of moduli of the surface with $B$ boundaries.

The counting of moduli becomes clearer if surfaces with at least one boundary component are described by the insertion of charges on a flat disk of unit radius in the $w$ plane (equivalently, the flat upper-half plane) instead of on the constant curvature sphere. A surface with $B$ boundaries is then described by the insertion of $B-1$ charges with independent strengths $\alpha_{i}$ at complex positions $w_{i}$ on a disk. Even though the action of the Mobius group $(S L(2, R)$ in this case) on (1) is again not a conformal transformation we know that three of these parameters must be redundant since the surface only has $3 B-6$ real moduli, so that the naive counting of parameters now works. From here on we will therefore insert charges on the flat disk, arbitrarily fixing one charge at the origin and another on the positive $w$ axis.

The electrostatic potential $\phi$ may be chosen to be the solution of the Laplace equation with sources (2) satisfying $\phi=\phi_{B}$ on the boundary of the disk, where $\phi_{B}$ is an arbitrary constant. This solution has the form

$$
\phi=\frac{1}{2}(\rho+\bar{\rho})
$$

where

$$
\rho \equiv \phi+i \psi=\sum_{i} \alpha_{i} \ln \left(\frac{w-w_{i}}{1-w \bar{w}_{i}}\right)
$$

The vector field is then given by

$$
A_{w}=-\frac{i}{2} \sum_{i} \frac{\alpha_{i}\left(1-w_{i} \bar{w}_{i}\right)}{\left(w-w_{i}\right)\left(1-w \bar{w}_{i}\right)}, \quad A_{\bar{w}}=\frac{i}{2} \sum_{i} \frac{\alpha_{i}\left(1-\bar{w}_{i} w_{i}\right)}{\left(\bar{w}-\bar{w}_{i}\right)\left(1-\bar{w} w_{i}\right)}
$$

The normal component of the vector potential vanishes on the boundary, i.e. $w A_{w}+$ $\bar{w} A_{\bar{w}}=A_{r}=0$ at $|w|=1$. 
The expression (11) defines a mapping from the disk to a string diagram in the style of Mandelstam [2] which is useful for describing the modified geometry. Infinitesimal circles around each of the points $w_{i}$ in the $w$ plane are mapped into incoming (when $\alpha_{i}>0$ ) or outgoing (when $\alpha_{i}<0$ ) closed strings of widths $2 \pi \alpha_{i}$ in $\psi$. If we choose (for convenience) all $\alpha_{i}>0$ the diagram represents a configuration in which $B-1$ closed strings enter at $\phi=-\infty$ and join together in pairs at the turning points, $\partial \rho / \partial w=0$, finally ending at the disk boundary, which is a closed string of width $2 \pi \alpha_{B}=-2 \pi \sum_{i=1}^{B-1} \alpha_{i}$ at $\phi=0$. The zeroes of the vector field (the turning points of this map) are mapped into the 'interaction' points where the cylinders join. In this mapping the $\alpha_{i}$ are the charges while in [2] they were components of the light-cone momenta of asymptotic string states.

The metric is diagonal in terms of the variables $\phi$ and $\psi$ defined by

$$
d \phi=i A_{w} d w-i A_{\bar{w}} d \bar{w}, \quad d \psi=A_{w} d w+A_{\bar{w}} d \bar{w}
$$

so that

$$
\begin{aligned}
d \tilde{s}^{2} & =d w d \bar{w}\left(1+2 A_{w} A_{\bar{w}}\right)+d w^{2} A_{w}^{2}+d \bar{w}^{2} A_{\bar{w}}^{2} \\
& =\frac{1}{4 A_{w} A_{\bar{w}}}\left(d \phi^{2}+\left(1+4 A_{w} A_{\bar{w}}\right) d \psi^{2}\right) \\
& =\left(1+\frac{d w}{d \rho} \frac{d \bar{w}}{d \bar{\rho}}\right)\left(d \phi^{2}\left(\frac{d \rho}{d w} \frac{d \bar{\rho}}{d \bar{w}}+1\right)^{-1}+d \psi^{2}\right) .
\end{aligned}
$$

The metric in the $\rho$ plane in the vicinity of the boundaries $\rho=\rho\left(w_{i}\right)$ has a simple form. Since $w-w_{i} \sim C_{i} e^{\rho / \alpha_{i}}$ (where $C_{i}=\left(1-w_{i} \bar{w}_{i}\right) \prod_{i \neq j}\left(\frac{w_{i}-w_{j}}{1-w_{i} \bar{w}_{j}}\right)^{-\alpha_{j} / \alpha_{i}}$ ) near $w=w_{i}$ (or $\phi=-\infty)$, the line element is approximately given by

$$
d \tilde{s}^{2} \sim \frac{d \phi^{2}}{\alpha_{i}^{2}} C_{i}^{2} e^{2 \phi / \alpha_{i}}+d \psi^{2}
$$

Changing variables from $\phi$ to $\tau$ on the $i$ th string in the vicinity of $\phi=-\infty$, where $\alpha_{i} d \tau \sim d \phi C_{i} e^{\phi / \alpha_{i}}$, gives

$$
\tau \sim C_{i} e^{\phi / \alpha_{i}}+D_{i}
$$

The $B-1$ boundaries at $w_{i}$ are at finite values of the $\tau$ variable determined by the parameters of the charges, whereas they are at $\phi=-\infty$.

For example, the two-boundary process is here described by a disk with a single charge at the origin in the $w$ plane. In this case $\rho \equiv \phi+i \psi=\alpha \ln w$ and the modified metric becomes

$$
d \tilde{s}^{2}=\left(e^{2 \phi / \alpha}+\alpha^{2}\right)\left(d \phi^{2} \frac{e^{2 \phi / \alpha}}{\alpha^{2}\left(e^{2 \phi / \alpha}+\alpha^{2}\right)}+d \sigma^{2}\right)
$$

(where $0 \leq \sigma \leq 2 \pi$ and $-\infty \leq \phi \leq 0$ ). Up to a (nonsingular) conformal factor this metric can be written as the flat metric on a cylinder by changing variables from $\phi$ 
to $\tau$, defined by $\tau=\sinh ^{-1}\left(\frac{e^{\phi / \alpha}}{|\alpha|}\right)$. The original world-sheet is therefore conformally equivalent to a cylinder of circumference $2 \pi$ and length $L=\sinh ^{-1}(1 /|\alpha|)$, which again becomes infinite in the limit $\alpha \rightarrow 0$ and is zero as $\alpha \rightarrow \infty$. The measure in $L(\alpha)$ translates into the measure in $\alpha$ according to

$$
d L=\frac{d \alpha}{|\alpha|}\left(\alpha^{2}+1\right)^{-1 / 2} \equiv f(\alpha) d \alpha
$$

The modified metric represents a world-sheet with holes of finite modulus, rather than punctures. A world-sheet with a puncture may be expressed by a metric of the form $d w d \bar{w} / w \bar{w}$ near $w=0$ and the world-sheet has an infinite snout located near $r=0$ where there is a curvature singularity. The integrated curvature, $\int d^{2} w \sqrt{g} R=4 \pi$ (where $g \equiv \operatorname{det} g$ ), appropriate to the insertion of an infinitesimal boundary, or puncture. By contrast the metric we are now considering has the form

$$
\frac{\left(2 w \bar{w}+\alpha^{2}\right) d w d \bar{w}}{2 w \bar{w}}-\frac{\alpha^{2} d w d w}{4 w^{2}}-\frac{\alpha^{2} d \bar{w} d \bar{w}}{4 \bar{w}^{2}}
$$

near $w=0$. The leading contribution to the curvature from the first term (the puncture term) is cancelled by the last two terms. The world-sheet is cylindrical and flat near the boundary with the curvature located on the interior of the sheet.

\section{Covering moduli space.}

In this section we would like to motivate our expectation that this parameterization covers moduli space. We start with the simplest nontrivial example, $B=3$, which we represent as in the previous section by the unit disk with two charges. Place $\alpha_{1}$ at the origin and $\alpha_{2}$ at $x<1$ on the positive $x$-axis, leaving three real parameters. It is easiest to consider first the corner of moduli space corresponding to three small holes. This is the same as three long cylinders (circumference $2 \pi$ and length $L_{i}$ ) joined together at one end and each having a boundary at the other. This limit corresponds to $x \rightarrow 0, \alpha_{1} / x \rightarrow 0, \alpha_{2} / x \rightarrow 0$. The lengths are then $L_{1} \sim \ln \left(x / \alpha_{1}\right), L_{2} \sim \ln \left(x / \alpha_{2}\right)$, and $L_{3} \sim \ln (1 / x)$. Restricting for now to positive charges gives a 6 -fold cover of moduli space in this region, from permuting the $L_{i}$. Equivalently, this a single cover of Teichmuller space, since the modular group is just the permutations. This might have been expected, since Teichmuller space is topologically $R^{3}$ and so is $\alpha_{1}-\alpha_{2}-x$ space, the natural ranges being $0<\alpha_{1}<\infty, 0<\alpha_{2}<\infty, 0<x<1$.

Now let us check that the rest of the boundary of Teichmuller space maps to the boundary of $\alpha_{1}-\alpha_{2}-x$ space. If, as seems plausible, the mapping has no folds in the interior, it then gives a single cover everywhere. There are three types of generic boundary: (i) One cylinder degenerating (becoming long). (ii) A strip degenerating, leaving an annulus with a thin strip attached across one boundary. (iii) A strip generating, leaving two annuli with a thin strip connecting a boundary from each. These are obtained 
in the $\alpha_{1}-\alpha_{2}-x$ parameterization as follows. In $1 . i$ the boundary $i$ is at the end of the degenerating cylinder ( $i=3$ is the boundary of the disk):

$$
\begin{aligned}
& \alpha_{1} \rightarrow 0, \quad \alpha_{2}, x \text { fixed } \\
& \alpha_{2} \rightarrow 0, \quad \alpha_{1}, x \text { fixed } \\
& x \rightarrow 0, \quad \alpha_{1} / x, \alpha_{2} / x \text { fixed }
\end{aligned}
$$

In $2 . i$, boundary $i$ is at the end of the annulus without the strip:

$$
\begin{aligned}
& \alpha_{2} \rightarrow \infty, \quad x, \alpha_{1} \text { fixed } \\
& \alpha_{1} \rightarrow \infty, \quad x, \alpha_{2} \text { fixed } \\
& x \rightarrow 0, \quad \alpha_{1}, \alpha_{2} \text { fixed } .
\end{aligned}
$$

In 3.i, boundary $i$ is pinching in the middle:

$$
\begin{aligned}
& x \rightarrow 0, \quad \alpha_{1}, \alpha_{2} / x \text { fixed } \\
& x \rightarrow 0, \quad \alpha_{2}, \alpha_{1} / x \text { fixed } \\
& x \rightarrow 1, \quad \alpha_{1}, \alpha_{2} /(1-x) \text { fixed }
\end{aligned}
$$

These limits can be understood by examining the metric in the region of the degeneration. Approaching the boundary of $\alpha_{1}-\alpha_{2}-x$ space in any other way corresponds to a multiple degeneration.

This extends readily to any $B$, giving a single cover of Teichmuller space or a $B$ !-fold cover of moduli space. In fact, it all works equally well for charges with any fixed set of signs. Allowing the charges to run from $-\infty$ to $\infty$ gives a $2^{B-1} B$ !-fold cover; the extra $2^{B-1}$ can be absorbed into the chemical potential for the charges.

One way of determining whether the parametrization of metrics in (1) really gives a single cover of moduli space might be to relate it to a standard parametrization, such as that given by the light-cone gauge. This is a parametrization in which world-sheet curvature is located at isolated "interaction" points (turning points of the boundaries), which correspond to the moduli of the surface. Given such a relation the measure for integrating over $\alpha_{i}$ and $w_{i}$ could be determined in terms of the integration measure in the light-cone parametrization. Experience with earlier string problems motivated by string quantum mechanics in the light-cone frame [2] suggests that the light-cone parameterization may also be useful for visualizing the physical effects of boundaries.

\section{Generating an arbitrary number of boundaries.}

The description of moduli space for a world-sheet with an arbitrary number of Dirichlet boundaries in terms of charges residing on a disk suggests a procedure for expressing the sum over all insertions in terms of a two-dimensional field theory on the disk. 
The integration over metrics in the functional integral that describes string theory on a genus zero world-sheet with $B$ boundaries reduces to integration over the $(3 B-6)$ dimensional moduli space for $B>2$ (1-dimensional for $B=2$ and 0-dimensional for $B<2)$. The fiducial metric may be chosen of the form $\tilde{g}_{\alpha \beta}=\delta_{\alpha \beta}+A_{\alpha} A_{\beta}$, where $A=-* d \phi$ is the potential for $B-1$ charges with arbitrary real strengths $\alpha_{i}$ at positions $w_{i}$ on the unit disk, satisfying $\partial^{2} \phi=\sum_{i=1}^{B-1} \kappa^{B-2} 2 \pi \alpha_{i} \delta\left(w-w_{i}\right)$. The integration over moduli is given by integration over $\alpha_{i}$ and $w_{i}$, so the string functional integral becomes (for $B>2$ )

$$
\begin{array}{r}
I_{B}=\kappa^{B-2} \int D X\left(\prod_{i=1}^{B-1} d^{2} w_{i} \sqrt{\tilde{g}\left(w_{i}\right)} d \alpha_{i}\right) f\left(\left\{\alpha_{i}\right\},\left\{w_{i}\right\}\right) \\
\exp \left(-\frac{1}{2 \pi} \int d^{2} w \sqrt{\tilde{g}} \tilde{g}^{\alpha \beta} \partial_{\alpha} X^{\mu} \partial_{\beta} X_{\mu}\right) .
\end{array}
$$

Here $\kappa$ is the closed-string coupling constant and the function $f$ is the measure on the parameter space of the charges that defines the measure on the moduli space of the surface with $B$ boundaries, and must be determined.

The configuration of charges can be obtained in the classical theory from an action of the form, $\frac{i}{2 \pi} \int d^{2} w\left(* F \chi-\sum_{i=1}^{B-1} \alpha_{i} \chi(w) \delta^{2}\left(w-w_{i}\right)\right)$. In this expression $* F=* d A=$ $-d * d \phi$ and the field $\chi$ is a Lagrange multiplier field with equations of motion that enforce the condition (2).

This suggests that (20) may be obtained from a functional integral on the disk with no holes so that the sum over boundaries would have the form,

$$
\begin{aligned}
\sum_{B=2}^{\infty} I_{B} & =\sum_{B=2}^{\infty} \kappa^{B-2} \int D \chi D \phi D X^{\mu} e^{\frac{-1}{2 \pi} \int d^{2} w \sqrt{g}\left(\tilde{g}^{\alpha \beta} \partial_{\alpha} X^{\mu} \partial_{\beta} X_{\mu}+i F \chi\right)} \\
& \int \prod_{i=1}^{B-1} f\left(\left\{\alpha_{i}\right\},\left\{w_{i}\right\}\right) d^{2} w_{i} \sqrt{\tilde{g}\left(w_{i}\right)} d \alpha_{i} e^{i \alpha_{i} \chi\left(w_{i}\right)} .
\end{aligned}
$$

More generally, $A_{\alpha}$ might be taken to be an independent vector field in which case the condition $A=-* d \phi$ arises from (2) in the gauge $* d A=0$.

It would be interesting to perform the sum in (21) explicitly so that the series may be reproduced by a functional integral based on an action that incorporates new world-sheet fields. As a schematic illustration of how this might work consider the action

$$
S=\frac{1}{2 \pi} \int d^{2} w\left(\sqrt{\tilde{g}} \tilde{g}^{\alpha \beta} \partial_{\alpha} X^{\mu} \partial_{\beta} X_{\mu}+i F \chi+\kappa \sqrt{\tilde{g}} \int d \alpha f(\alpha) \cos \alpha \chi\right)
$$

(where $\tilde{g}$ is of the form (1)). Expanding the functional integral $\kappa^{-1} \int D g D X D \chi D \phi e^{-S}$ in powers of $\kappa$ produces an expansion in increasing number of charge insertions and hence of Dirichlet boundaries. Thus, in the $O\left(\kappa^{-1}\right)$ term the Lagrange multiplier $\chi$ enforces 
the condition $F=0$ that sets $\phi=0$, leaving the usual string functional on a disk. The $O\left(\kappa^{0}\right)$ term gives rise to the $B=2$ term in (21). Higher-order terms lead to expressions like (21) with a particular form for $f$. Note that the exponential brings in a factor $1 /(B-1)$ !, so this over-covers moduli space by a factor $B$ : the outer boundary of the disk must be treated as distinguished. Of course, the full measure involves a complicated Fadeev-Popov determinant; it can take a local form such as (22) when this is represented in terms of ghost fields. The mapping of the boundary to a point, while very natural for the $X^{\mu}$, is less simple for the ghosts; this may be an obstacle for the idea that we are proposing in this section.

Perhaps the details should not at present be taken seriously, but this suggests the possibility of expressing the sum over Dirichlet boundaries in terms of a stringy action that incorporates some novel features. The strong similarity of the modified metric to that arising in Kaluza-Klein compactification suggests that the sum over boundaries might be naturally expressed in terms of an action principal on a three-dimensional worldsheet. Given such a formulation the hope would be that instead of expanding in powers of $\kappa$, non-perturbative information may be deduced by a mean field approximation.

Everything described explicitly in this paper refers to boundary corrections to closed-string tree diagrams, thereby defining a modified closed-string theory. The generalization to world-sheets of higher genus (i.e., with handles), is straightforward in principle, thus defining the theory in which there is a condensate of Dirichlet boundaries in every order in closed-string perturbation theory. Unlike conventional string theories this is known to have power-behaved fixed-angle cross-sections. A formulation of an effective theory, such as that illustrated earlier, should be of interest in determining how the Dirichlet boundary condensate affects the massless spectrum.

\section{Acknowledgments}

M.B.G. is grateful to Orlando Alvarez for discussions, and wishes to thank the Miller

Institute, Berkeley, for support as a Visiting Miller Professor during September 1993. The work of J. P. was supported by NSF grants PHY-89-04035 and PHY-91-16964. 


\section{REFERENCES}

1. M.B. Green, Space-Time duality and Dirichlet string theory, Phys. Lett. 266B (1991) 325.

2. S. Mandelstam, Interacting-string picture of dual resonance models, Nucl. Phys. B64 (1973) 205. 